Pacific Journal of Mathematics

REFLECTION OF BIHARMONIC FUNCTIONS ACROSS
ANALYTIC BOUNDARY CONDITIONS WITH EXAMPLES 


\section{REFLECTION OF BIHARMONIC FUNCTIONS ACROSS ANALYTIC BOUNDARY CONDITIONS WITH EXAMPLES}

\section{JAMES M. SLOSS}

1. Introduction. This paper is concerned with the reflection of solutions $u$ of the biharmonic equation $\Delta^{2} u=0$ across an analytic arc $\gamma$ when $u$ satisfies on $\gamma$ two linear analytic boundary conditions. If the coefficients are subject to proper regularity conditions, then the region into which $u$ can be extended is dependent only on the analytic arc and the original region on which $u$ is defined; i.e., it is dependent only on geometric quantities and therefore is what may be called "reflection in the large." The case in which the boundary conditions are nonlinear was treated in [3], but extension in that case is only local.

We consider two boundary conditions whose independence is stated by an inequality. It is shown that this inequality is satisfied automatically in the case of the first, second, and mixed fundamental boundary value problems of elasticity.

Finally, we shall give applications of the first boundary value problem, in which case the reflection is effected by quadratures, for a number of special geometrical configurations. In these cases the reflection of $u$ can be expressed explicitly.

2. Reflection across an analytic arc. Let the open analytic arc $\gamma$ be defined by the real analytic relation $F(x, y)=0$ where, for every point $(x, y)$ on $\gamma, F_{x}(x, y) \neq 0$ or $F_{y}(x, y) \neq 0$.

Let $\zeta=\xi+i \eta$ and $z=x+i y$ be two complex variables and consider:

$$
g(z, \zeta) \equiv F\left[\frac{z+\zeta}{2}, \frac{z-\zeta}{2 i}\right]=0
$$

where $g(z, \zeta)$ is an analytic function of $(z, \zeta)$ in a polycylindrical neighborhood of $\left(z_{0}, \bar{z}_{0}\right)$ for every $z_{0}$ on $\gamma$. For $z_{0}=x_{0}+i y_{0}$ on $\gamma$ :

$$
g\left(z_{0}, \bar{z}_{0}\right)=F\left(x_{0}, y_{0}\right)=0
$$

and, for $(z, \zeta)=\left(z_{0}, \bar{z}_{0}\right)$,

$$
\frac{\partial g}{\partial \zeta}\left(z_{0}, \bar{z}_{0}\right)=\left(\frac{1}{2}\right)\left\{F_{x}\left(x_{0}, y_{0}\right)+i F_{y}\left(x_{0}, y_{0}\right)\right\} \neq 0, \frac{\partial g}{\partial z}=\overline{\left(\frac{\partial g}{\partial z}\right)} \text {. }
$$

Received August 23, 1962. The author wishes to acknowledge support by the Office of Naval Research Contract Nonr 222 (62) (Nr 041-214). The author wishes to express his appreciation to Hans Lewy for his encouragement on this problem. 
There exists, by the implicit function theorem of complex variables, a unique function: $\zeta=G(z)$ which establishes a one-to-one correspondence between points $z$ in a neighborhood of $z_{0}$, and points $\zeta$ in a neighborhood of $\bar{z}_{0}$ and for which $G\left(z_{0}\right)=\bar{z}_{0}$. Moreover, $G(z)$ is analytic in a neighborhood of $\gamma$.

Consider the function $\hat{z}=\overline{G(z)}$ and call the point $\hat{z}$ the reflection of $z$ across $\gamma$. For $z$ on $\gamma, \hat{z}=z$.

Consider a semi-neighborhood $\Omega$ of $\gamma$ in which $G(z)$ is analytic and univalent so that $G^{\prime}(z) \neq 0$ in $\Omega$.

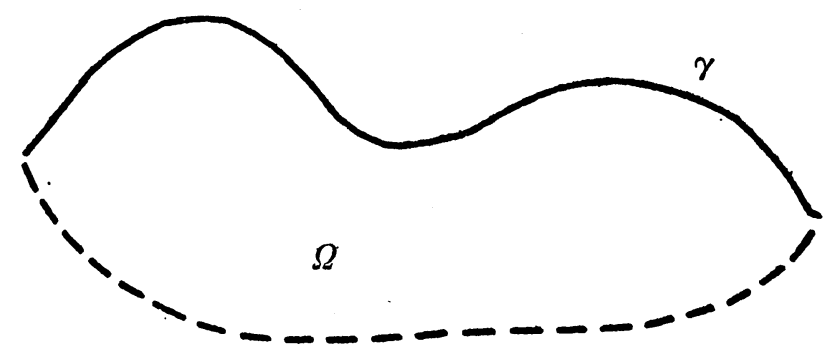

Denote $\overline{G(\Omega)}$ by $\hat{\Omega}$ and assume $\hat{\Omega} \cap \Omega$ empty; set $\bar{z}=H(\hat{z}), \hat{z}$ in: $\hat{\Omega} \cup \gamma$. Then $H(\hat{z})$ is analytic for $\hat{z}$ in $\hat{\Omega}$ and agrees with $G(\hat{z})$ for $\widehat{z}$ on $\gamma$. Thus by analytic continuation, $H(\hat{z})=G(\hat{z})$ for $\hat{z}$ in $\hat{\Omega} \cup \gamma$. Finally, since $\widehat{z}=\overline{G(z)}, z$ in $\Omega \cup \gamma$, we have

$$
\widehat{z}=\overline{G[\overline{G(z)}]}=z, \quad z \text { in } \Omega \cup \gamma
$$

and as easily verified, for $z$ in $\Omega \cup \gamma \cup \hat{\Omega}$. Thus $\Omega=\overline{G(\hat{\Omega})}, G$ is: univalent in $\hat{\Omega}$ and $G^{\prime}(z) \neq 0$ in $\hat{\Omega}$.

We shall need later the fact that the direction cosines of a normal to $\gamma$ can be extended to analytic functions of $z$ on $\Omega \cup \gamma \cup \hat{\Omega}$. To. this end let

$$
\begin{aligned}
& \alpha(z)=\cos (t, x) \quad \text { for } z \text { on } \gamma \\
& \beta(z)=\cos (t, y)
\end{aligned}
$$

where $t=$ tangent to $\gamma$. Then on $\gamma, \bar{z}=G(z)$, thus differentiation with respect to arc length $s$ on $\gamma$ yields

$$
\alpha-i \beta=G^{\prime}(z) \frac{d z}{d s}=G^{\prime}(z)(\alpha+i \beta) .
$$

Furthermore

$$
(\alpha+i \beta)(\alpha-i \beta)=1 \text { on } \gamma .
$$

Thus:

$$
\alpha-i \beta=\sqrt{ } G^{\prime}(z) ; \quad \alpha+i \beta=1 / \sqrt{ } G^{\prime}(z)
$$


where the square root is uniquely defined on $\gamma$. These definitions of $\alpha$ and $\beta$ now uniquely determine the analytic continuation of $\alpha$ and $\beta$ into $\Omega$ which is simply connected, and its image $\hat{\Omega}$ by reflection across $\gamma$.

3. Canonical representation of a biharmonic function. A function $u(x, y)$ is biharmonic on a simply connected open set $A$ if $\Delta u$ is harmonic there. We follow Muskhelishvili [2] in obtaining a representation of a biharmonic function on $\Omega$.

Let $v(x, y)$ be a conjugate function of $\Delta u$ for $(x, y)$ in $\Omega$. Then $\Delta u+i v$ is an analytic function for $(x, y)$ in $\Omega$ and therefore the line integral $\int_{z_{0}}^{z}[\Delta u+i v] d z$ whose path remains in $\Omega$, is independent of the path and

$$
\varphi(z) \equiv \varphi_{1}(x, y)+i \varphi_{2}(x, y)=\frac{1}{4} \int_{z_{0}}^{z}[\Delta u+i v] d z, \quad\left(z_{0}, z \text { in } \Omega\right)
$$

represents a function analytic for $z$ in $\Omega$.

Let

$$
\psi_{1}(x, y)=u-\operatorname{Re}\{\bar{z} \varphi(z)\}
$$

and note that $\Delta \psi_{1}=0$. Therefore, $\psi_{1}(x, y)$ is an harmonic function in $\Omega$. Let $\psi_{2}(x, y)$ be a conjugate harmonic function to $\psi_{1}(x, y)$ then $\psi(z) \equiv \psi_{1}(x, y)+i \psi_{2}(x, y)$ is analytic in $\Omega$ and

$$
2 u(x, y)=\bar{z} \varphi(z)+z \overline{\varphi(z)}+\psi(z)+\overline{\psi(z)}
$$

where $\varphi(z)$ and $\psi(z)$ are analytic for $z$ in $\Omega$.

Set

$$
\begin{aligned}
& \varphi^{*}(z)=\overline{\varphi(\widehat{z})}=\overline{\varphi[\overline{G(z)}]}, \quad \text { for } z \text { in } \hat{\Omega} \text {, } \\
& \psi^{*}(z)=\overline{\psi(\hat{z})}=\overline{\psi[\overline{G(z)}]} \text {, for } z \text { in } \hat{\Omega} \text {. }
\end{aligned}
$$

Then $\varphi^{*}(z)$ and $\psi^{*}(z)$ are analytic functions for $z$ in $\hat{\Omega}$.

If we replace $z$ by $\widehat{z},(\hat{z})$ by $z$ in (3.3), substitute (3.3) into (3.2) and note that $\bar{z}=G(\widehat{z})$ we find

$$
2 u(x, y)=G(\widehat{z}) \varphi(z)+z \varphi^{*}(\widehat{z})+\psi(z)+\psi^{*}(\widehat{z}) \text { for }(x, y) \text { in } \Omega .
$$

This yields a representation of $u(x, y)$ in terms of two functions, $\varphi(z)$ and $\psi(z)$, analytic for $z$ in $\Omega$, and two functions, $\varphi^{*}(z)$ and $\psi^{*}(z)$, analytic for $z$ in $\hat{\Omega}$, the reflection of $\Omega$ across $\gamma$.

4. Reflection of biharmonic functions across linear analytic boundary conditions. With the notations of $\S \S 2$ and 3 and $u_{j_{n}}=(\partial / \partial x)^{j}(\partial / \partial y)^{n} u$, $0 \leqq j ; 0 \leqq n$, we state: 
THEOREM 1. Let $3 \geqq p_{1} \geqq p_{2} \geqq 0, p=\max \left\{2, p_{1}\right\}$ and let $u(x, y)$ be a function biharmonic on $\Omega$, in $C^{p}(\Omega \cup \gamma)$ and let the conjugate harmonic functions of $\Delta u$ belong to $C^{p-2}(\Omega \cup \gamma)$. Let $u(x, y)$ satisfy on $\gamma$ the two linear conditions:

$$
\begin{aligned}
\sum_{j+n=0}^{p_{m}} A_{j n}^{m}(z) u_{j n}(x, y)+B_{1}^{m}(z) \varphi_{1}(x, y)+B_{2}^{m}(z) \varphi_{2}(x, y)=f^{m}(z) & ; \\
m & =1,2
\end{aligned}
$$

where $p_{m}=0$ presupposes $B_{j}^{m}=0$ and where $A_{j n}^{m}(z), B_{j}^{n}(z)$ and $f^{m}(z)$ are analytic functions for $z$ in $\Omega \cup \gamma \cup \hat{\Omega}$. Assume that the following inequality for the coefficients of (I) holds.

$$
\begin{aligned}
0 \neq\left(p_{1}-p_{2}\right) G^{\prime}(z)\left\{\sum_{j+n=p_{1}}(i)^{n} A_{j n}^{1}\right\}\left\{\sum_{j+n=p_{2}}(i)^{n} A_{j n}^{2}\right\} \\
+\left|\begin{array}{l}
\sum_{j+n=p_{1}}(i)^{n} A_{j n}^{1}(z) ;\left\{\sum_{j+n=p_{1}}(i)^{n}(j-n) A_{j n}^{1}(z)+\delta_{0}^{p_{1}-1}\left[B_{1}^{1}(z)-i B_{2}^{1}(z)\right]\right\} \\
\sum_{j+n=p_{2}}(i)^{n} A_{j n}^{2}(z) ;\left\{\sum_{j+n=p_{2}}(i)^{n}(j-n) A_{j n}^{2}(z)+\delta_{0}^{p_{2}-1}\left[B_{1}^{2}(z)-i B_{2}^{2}(z)\right]\right\}
\end{array}\right|
\end{aligned}
$$

for $z$ in $\Omega \cup \gamma \cup \hat{\Omega}$ where $\delta_{j}^{k}=1$ if $j=k$ and $=0$ if $j \neq k$. Then we can reflect $u(x, y)$ across $\gamma$ into $\hat{\Omega}$ i.e. there exists a unique function $u(x, y)$ which is biharmonic in $\Omega \cup \gamma \cup \hat{\Omega}$ and which agrees with the given $u$ in $\Omega \cup \gamma$.

Note that $p_{1} \geqq 1$ since if $p_{1}=p_{2}=0$ then condition (II) is never satisfied.

Condition II is natural, as it merely insures the independence of the two boundary conditions.

We shall give an outline of the proof for the general theorem. We shall then prove the theorem in detail for the special case of the first boundary value problem of elasticity, since most essential points. of the general proof appear in the special case, but the computations. are considerably shorter.

\section{Proof outline.}

4.1. From the continuity requirements on $u$ and $v$ it is shown that $\varphi(z)$ and $\psi(z)$ defined in $\S 3$ belong to $C^{p-1}(\Omega \cup \gamma)$.

4.2. A representation of the derivatives of $u, \varphi_{1}$ and $\varphi_{2}$, in terms. of $\varphi(z), \psi(z), \varphi^{*}(\hat{z})$ and $\psi^{*}(\hat{z})$ is derived for $z$ in $\Omega$.

4.3. Because of the continuity conditions on $u$, we express the highest order derivatives of $u$ with respect to $x$ and $y$, that occur in the boundary condition, in terms of the tangential derivative along $\gamma$ of the lower order derivatives of $u$.

4.4. The derivatives of $u$, evaluated on $\gamma$, can be linearly expressed in terms of two new functions $P(z)$ and $\Phi(z)$ which are analytic 
in $\Omega$ and continuous in $\Omega \cup \gamma$, and two other functions $P^{*}(z)$ and $\Phi^{*}(z)$ which are analytic in $\hat{\Omega}$ and continuous in $\hat{\Omega} \cup \gamma$.

A. We introduce for the first two functions

$$
\begin{array}{ll}
P(z)=G(z) \varphi^{\left(p_{1}\right)}(z)+\psi^{\left(p_{1}\right)}(z), & \text { for } z \text { on } \Omega, \\
\Phi(z)=\varphi^{\left(p_{1}-1\right)}(z), & \text { for } z \text { on } \Omega \cup \gamma .
\end{array}
$$

$\Phi(z)$ and $P(z)$ are analytic for $z$ in $\Omega, \Phi(z)$ is continuous for $z$ in $\Omega \cup \gamma$ by 1 ; it is proved that also $P(z)$ can be continuously extend to $\Omega \cup \gamma$.

B. For the other two functions we introduce

$$
\begin{array}{ll}
P^{*}(z)=z \varphi^{*\left(p_{1}\right)}(z)+\varphi^{*\left(p_{1}\right)}(z), & \text { for } z \text { on } \hat{\Omega} \\
\Phi^{*}(z)=\varphi^{*\left(p_{1}-1\right)}(z), & \text { for } z \text { on } \hat{\Omega} \cup \gamma .
\end{array}
$$

It is seen that $\Phi^{*}(z)$ and $P^{*}(z)$ are both analytic for $z$ in $\hat{\Omega}, \Phi^{*}(z)$ is: continuous in $\hat{\Omega} \cup \gamma$ by $\S 1$ and $P^{*}(z)$ is continuously extensible to. $\hat{\Omega} \cup \gamma$.

4.5. Both boundary conditions can now be expressed in terms of $P(z), \Phi(z), P^{*}(z)$ and $\Phi^{*}(z)$. By differentiation, if necessary, of the second boundary condition (I.2), and by use of condition II we can solve the two equations for $P(z)$ and $\Phi(z)$ and thus put the boundary conditions in the form:

$$
\begin{aligned}
& P(z)=C_{1}(z)+\int_{z_{0}^{\gamma}}^{z} d t F_{11}(z, t) P(t)+\int_{z_{0}^{\gamma}}^{z} d t F_{12}(z, t) \Phi(t) \\
& \Phi(z)=C_{2}(z)+\int_{z_{0}^{\gamma}}^{z} d t F_{21}(z, t) P(t)+\int_{z_{0}^{\gamma}}^{z} d t F_{22}(z, t) \Phi(t)
\end{aligned}
$$

for $z$ and $z_{0}$ on $\gamma$, where $C_{j}(z)$ are certain functions analytic for $z$ in $\hat{\Omega}$ and continuous for $z$ in $\hat{\Omega} \cup \gamma$ and $F_{j k}(z, t)$ are functions analytic in $z$ and $t$ for $z$ and $t$ on $\Omega \cup \gamma \cup \hat{\Omega}$. Because of the continuity restrictions on $u$ there are four cases:

Case 1. $p_{1}=p_{2}=1$.

Case 2. $p_{1}=p_{2} \geqq 2$.

Case 3. $p_{1}=1, p_{2}=0$, and

Case 4. $p_{1} \geqq 2, p_{1}>p_{2} \geqq 0$.

4.6. The equations (4.5.0), which hold on $\gamma$, are considered as Volterra integral equations for $z$ in the whole of $\hat{\Omega} \cup \gamma$. These equations have unique solutions $P_{e x}(z)$ and $\Phi_{e x}(z)$ which are continuous for $z$ in $\hat{\Omega} \cup \gamma$ and analytic for $z$ in $\hat{\Omega}$.

4.7. Since $P_{e x}(z)$ and $\Phi_{e x}(z)$ satisfy on $\gamma$ the same equations as $P(z)$ and $\Phi(z)$ and since on $\gamma$ there can be only one such set of func- 
tions, $P_{e x}(z)=P(z)$ and $\Phi_{e x}(z)=\Phi(z)$ on $\dot{\gamma}$. Since $P_{e x}(z)$ and $\Phi_{e x}(z)$ are continuous for $z$ in $\hat{\Omega} \cup \gamma, P_{e x}(z)$ and $\Phi_{e x}(z)$ analytically continue $P(z)$ and $\Phi(z)$ into $\hat{\Omega} \cup \gamma$.

4.8. The final step is the observation that the analytic extension of the auxiliary functions $P(z)$ and $\Phi(z)$ yields the analytic extension of $\varphi(z)$ and $\psi(z)$ and thereby the extension of $u$.

5. Applications of Theorem 1 with proof of application 2. We shall show that Theorem 1 applies to what Muskhelishvili [2] has called the first and second fundamental boundary value problems of elasticity.

Application 1 (Second boundary value problem). Let $u(x, y)$ be a function which is biharmonic on $\Omega$, and let $u(x, y) \in C^{2}(\Omega \cup \gamma)$ and let the conjugates to $\Delta u$ belong to $C(\Omega \cup \gamma)$. Then, if the given displacement vector $\left[f^{1}(z), f^{2}(z)\right]$ has both components analytic in $\Omega \cup \gamma \cup \hat{\Omega}$ and if on $\gamma$ :

$$
\begin{aligned}
& f^{1}(z)=-\frac{1}{2 \mu} u_{x}+\frac{\lambda+2 \mu}{(\lambda+\mu) \mu} \operatorname{Re} \varphi \\
& f^{2}(z)=-\frac{1}{2 \mu} u_{y}+\frac{\lambda+2 \mu}{(\lambda+\mu) \mu} \operatorname{Im} \varphi
\end{aligned}
$$

where $\lambda$ and $\mu$ are the Lamé coefficients, then $u(x, y)$ can be continued biharmonically across $\gamma$ into $\hat{\Omega}$.

Proof. Condition II of Theorem 1 is the only condition which needs to be checked. Since $p_{1}=p_{2}=1$, condition II becomes:

$$
\begin{aligned}
0 & \neq\left|\begin{array}{ll}
A_{10}^{1}+i A_{01}^{1} ; & A_{10}^{1}-i A_{01}^{1}+B_{1}^{1}-i B_{2}^{1} \\
A_{10}^{2}+i A_{01}^{2} ; & A_{10}^{2}-i A_{01}^{2}+B_{1}^{2}-i B_{2}^{2}
\end{array}\right| \\
& =\left|\begin{array}{cc}
-\frac{1}{2 \mu} ; \quad-\frac{1}{2 \mu}+\frac{\lambda+2 \mu}{(\lambda+\mu) \mu} \\
-i \frac{1}{2 \mu} ; \quad+i \frac{1}{2 \mu}-i \frac{\lambda+2 \mu}{(\lambda+\mu) \mu}
\end{array}\right|=\frac{\lambda+3 \mu}{2 \mu^{2}(\lambda+\mu)} i .
\end{aligned}
$$

The condition is satisfied since $\lambda>0$ and $\mu>0$.

Application a (First boundary value problem) Let $u(x, y)$ be the Airy stress function which is biharmonic on $\Omega$ and satisfies along $\gamma$ :

$$
\begin{aligned}
& X_{n}(z)=-u_{x y} \cos (n, y)+u_{y y} \cos (n, x) \\
& Y_{n}(z)=u_{x x} \cos (n, y)-u_{x y} \cos (n, x)
\end{aligned}
$$

where $\left[X_{n}(z), Y_{n}(z)\right]$ is the force per unit arc length of $\gamma$ acting in 
the direction of the normal $n$ to $\gamma$. Let $X_{n}(z)$ and $Y_{n}(z)$ be functions which are analytically extensible for $z$ in $\Omega \cup \gamma \cup \hat{\Omega}$. Let $u \in C^{2}(\Omega \cup \gamma)$ and the harmonic conjugates of $\Delta u$ belong to $C(\Omega \cup \gamma)$. Then we can extend $u(x, y)$ biharmonically into $\Omega \cup \gamma \cup \hat{\Omega}$.

Proof: If we assume $n$ and $t$ are oriented in the same way as $x$ and $y$ then $(n, y)=(t, x) \pm \pi,(n, x)=(t, y)$ and thus $\cos (t, x)=$ $-\cos (n, y)$ and $\cos (t, y)=\cos (n, x)$.

Now the boundary conditions on $\gamma$ can be written

$$
\begin{aligned}
& X_{n}(z)=u_{x y} \alpha(z)+u_{y y} \beta(z) \\
& Y_{n}(z)=-u_{x x} \alpha(z)-u_{x y} \beta(z) .
\end{aligned}
$$

Condition II of the theorem becomes then

$$
\begin{aligned}
\left|\begin{array}{ll}
A_{20}^{1}+i A_{11}^{1}-A_{02}^{1} ; 2 A_{20}^{1}+2 A_{02}^{1} \\
A_{20}^{2}+i A_{11}^{2}-A_{02}^{2} ; 2 A_{20}^{2}+2 A_{02}^{2}
\end{array}\right| & =\left|\begin{array}{l}
i \alpha(z)-\beta(z) ; 2 \beta(z) \\
-\alpha(z)-i \beta(z) ;-2 \alpha(z)
\end{array}\right| \\
& =-2 i(\alpha+i \beta)^{2} \neq 0
\end{aligned}
$$

which is satisfied for $z$ in $\Omega \cup \gamma \cup \hat{\Omega}$.

We now take up the details of the proof.

4.1. $\Delta u$ and its conjugates are in $C(\Omega \cup \gamma)$. Therefore from (3.0) we see that

$$
\varphi_{1 x}(x, y)+i \varphi_{2 x}(x, y)=\frac{1}{4}[\Delta u+i v] \text { for } z \text { in } \Omega
$$

and therefore $\varphi_{1 x}=\varphi_{2 y}$ and $\varphi_{2 x}=-\varphi_{1 y}$ are continuous for $z$ in $\Omega \cup \gamma$ i.e. $\varphi(z) \in C^{1}(\Omega \cup \gamma)$. From (3.1) we see that $\psi_{1}(x, y) \in C^{1}(\Omega \cup \gamma)$ and therefore by the Cauchy-Riemann conditions, $\psi_{2}(x, y)$ and also $\psi(z) \in$ $C^{1}(\Omega \cup \gamma)$. From (3.3) and the fact that $G(z)$ is analytic on $\Omega \cup \gamma \cup \hat{\Omega}$ we see that $\varphi^{*}(z)$ and $\psi^{*}(z)$ belong to $C^{1}(\hat{\Omega} \cup \gamma)$.

4.2. For $u$ and its derivatives in terms of $\varphi(z), \psi(z), \varphi^{*}(z)$ and $\psi^{*}(z)$ we have for $z \in \Omega$ :

$$
\begin{aligned}
& 2 u(x, y)=\bar{z} \varphi(z)+\psi(z)+z \varphi^{*}[\overline{G(z)}]+\psi^{*}[\overline{G(z)}] \\
& =G(\widehat{z}) \varphi(z)+\psi(z)+z \varphi^{*}(\widehat{z})+\psi^{*}(\widehat{z}), \\
& 2 D_{x} u=\varphi(z)+G(\hat{z}) \varphi^{\prime}(z)+\psi^{\prime}(z)+\varphi^{*}(\hat{z}) \\
& +z \varphi^{* \prime}(\widehat{z}) \overline{G^{\prime}(z)}+\psi^{* \prime}(\widehat{z}) \overline{G^{\prime \prime}(z)} \text {, } \\
& 2 D_{v} u=-i \varphi(z)+i G(\hat{z}) \varphi^{\prime}(z)+i \psi^{\prime}(z) \\
& -i\left[z \varphi^{* \prime}(\widehat{z})+\psi^{* \prime}(\widehat{z})\right] \overline{G^{\prime}(z)}+i \varphi^{*}(\widehat{z}), \\
& 2 D_{x x} u=G(\hat{z}) \varphi^{\prime \prime}(z)+\psi^{\prime \prime}(z)+2 \varphi^{\prime}(z) \\
& +\left[z \varphi^{* \prime \prime}(\widehat{z})+\psi^{* \prime \prime}(\widehat{z})\right]\left[\overline{G^{\prime}(z)}\right]^{2}+\cdots,
\end{aligned}
$$




$$
\begin{aligned}
2 D_{x y} u= & i G(\hat{z}) \varphi^{\prime \prime}(z)+i \psi^{\prime \prime}(z) \\
& -i\left[z \varphi^{* \prime \prime}(\widehat{z})+\psi^{* \prime \prime}(\widehat{z})\right]\left[\overline{G^{\prime}(\widehat{z})}\right]^{2}+\cdots, \\
2 D_{y y} u= & -G(\hat{z}) \varphi^{\prime \prime}(z)-\psi^{\prime \prime}(z)+2 \varphi^{\prime}(z) \\
& -\left[z \varphi^{* \prime \prime}(\widehat{z})+\psi^{* \prime \prime}(\widehat{z})\right]\left[\overline{G^{\prime}(z)}\right]^{2}+\cdots,
\end{aligned}
$$

where $+\cdots$ is to be read "plus lower order derivatives of $\varphi^{*}(\hat{z})$ and $\psi^{*}(\widehat{z}) . "$

4.3. The first order derivatives of $u$ on $\gamma$ are thus expressed in terms of functions continuous on $\Omega \cup \gamma$. As for the second derivatives, we consider the tangential derivative of $u$ along $\gamma$

$$
D_{t} u=D_{s} u=\frac{\partial u}{\partial x} \frac{d x}{d s}+\frac{\partial u}{\partial y} \frac{d y}{d s}
$$

where $s=\operatorname{arc~length~of~} \gamma$. Therefore

$$
D_{t} u=D_{x} u \cos (t, x)+D_{y} u \cos (t, y)=\alpha(z) D_{x} u+\beta(z) D_{y} u \text {. }
$$

Now set for $z$ in $\Omega \cup \gamma$ :

$$
\widetilde{D}_{t}=\alpha(z) D_{x}+\beta(z) D_{y} .
$$

Then for a function $f \in C^{1}, \lim _{z \rightarrow \gamma} \widetilde{D}_{t} f=D_{t} f$.

For $z \in \Omega$

$$
\begin{aligned}
\widetilde{D}_{t} D_{z}\{2 u\}= & \left(\alpha D_{x}+\beta D_{y}\right)(1 / 2)\left(D_{x}-i D_{y}\right) \\
& \cdot\{\bar{z} \varphi(z)+\psi(z)+z \overline{\varphi(z)}+\overline{\psi(z)}\} \\
= & {\left[\bar{z} \varphi^{\prime \prime}(z)+\psi^{\prime \prime}(z)\right](\alpha+i \beta)+\left[\varphi^{\prime}(z)+\overline{\varphi^{\prime}(z)}\right](\alpha-i \beta) } \\
= & {\left[G(\hat{z}) \varphi^{\prime \prime}(z)+\psi^{\prime \prime}(z)\right][\alpha(z)+i \beta(z)] } \\
& \left.\left.+\left[\varphi^{\prime}(z)+\varphi^{* \prime}(\hat{z}) \overline{G^{\prime}(z)}\right]\right] \alpha(z)-i \beta(z)\right] .
\end{aligned}
$$

Likewise for $z$ in $\Omega$

$$
\widetilde{D}_{t} D_{\bar{z}}\{2 u\}=\left(\alpha D_{x}+\beta D_{y}\right)(1 / 2)\left(D_{x}+i D_{y}\right)
$$

$$
\begin{aligned}
& \cdot\left\{\bar{z} \varphi(z)+\psi(z)+z \varphi^{*}[\overline{G(z)}]+\psi^{*}[\overline{G(z)}]\right\} \\
& =\left[z \varphi^{* \prime \prime}(\hat{z})+\psi^{* \prime \prime}(\hat{z})\right]\left[\overline{G^{\prime}(z)}\right]^{2}[\alpha(z)-i \beta(z)]
\end{aligned}
$$$$
+\varphi^{\prime}(z)[\alpha+i \beta]+\text { starred terms of order less than } 2 \text {. }
$$

Since $\alpha+i \beta \neq 0$ and $\alpha-i \beta \neq 0$ for $z$ in $\Omega \cup \gamma \cup \hat{\Omega}$ we can utilize (4.3.1) and (4.3.2) to express the second derivatives of $u$ for $z \in \Omega$ from (4.2.1), (4.2.2) and (4.2.3) as: 


$$
\begin{aligned}
2 D_{x}^{j} D_{y}^{n} u=(i)^{n}\left\{\frac{2}{\alpha+i \beta} \widetilde{D}_{t} D_{z} u+(j-n) \varphi^{\prime}(z)\right. \\
\left.\quad-\frac{\alpha-i \beta}{\alpha+i \beta}\left[\varphi^{\prime}(z)+\varphi^{* \prime}(\hat{z}) \overline{G^{\prime}(z)}\right]\right\} \\
+(-i)^{n}\left\{\frac{2}{\alpha-i \beta} \widetilde{D}_{t} D_{\bar{z}} u-\frac{\alpha+i \beta}{\alpha-i \beta} \varphi^{\prime}(z)+\cdots\right\}
\end{aligned}
$$

where $j+n=2$ and $+\cdots$ means derivatives of $\varphi^{*}(z)$ and $\psi^{*}(z)$ of order less than 2 . Since $u \in C^{2}(\Omega \cup \gamma)$, both sides remain continuous for $z$ in $\Omega \cup \gamma$ and therefore (4.3.3) is valid not only in $\Omega$ but in $\Omega \cup \gamma$.

4.4.A. From (4.1) we see that $\Phi(z) \equiv \varphi^{\prime}(z)$ is analytic for $z$ in $\Omega$ and continuous in $\Omega \cup \gamma$. It is clear that

$$
P(z)=G(z) \varphi^{\prime \prime}(z)+\psi^{\prime \prime}(z)
$$

is analytic for $z$ in $\Omega$. To show that $P(z) \in C(\Omega \cup \gamma)$ we consider

$$
Q(z) \equiv G(z) \varphi^{\prime}(z)+\psi^{\prime}(z) \text {. }
$$

Since $\widetilde{D}_{t} D_{z}\{2 u\} \in C(\Omega \cup \gamma)$ and $\widetilde{D}_{t} D_{z}\{2 u\}=D_{s} D_{z}\{2 u\}$ on $\gamma$ where $s=$ are length of $\gamma$, we have for $z \in \gamma$ :

$$
\begin{aligned}
\int_{z_{0}^{\gamma}}^{z} D_{s} D_{z}\{2 u\} d s & \left.=D_{z} 2 u\right]_{z_{0}}^{z}=\left[\bar{z} \varphi^{\prime}(z)+\psi^{\prime}(z)+\overline{\varphi(z)}\right]_{z_{0}}^{z} \\
& =Q(z)-Q\left(z_{0}\right)+\overline{\varphi(z)}-\overline{\varphi\left(z_{0}\right)} .
\end{aligned}
$$

Therefore for $z$ on $\gamma$, we find:

$$
Q(z)=2 \int_{z_{0}^{\gamma}}^{z} D_{s} D_{z} u d s-\varphi^{*}(z)+\text { constant },
$$

and (4.4.2) $D_{t} Q(z)=2 D_{t} D_{z} u-(\alpha+i \beta) \varphi^{* \prime}(z)$.

Thus $D_{t} Q(z) \in C(\gamma)$. Also $Q(z) \in C(\Omega \cup \gamma)$. As seen in the appendix it follows that $Q^{\prime}(z)$ is continuous in $\Omega \cup \gamma$. Since for $z \in \Omega$ :

$$
\widetilde{D}_{t} Q(z)=\left(\alpha D_{x}+\beta D_{y}\right) Q(z)=(\alpha+i \beta) Q^{\prime}(z) .
$$

This formula holds in $\Omega \cup \gamma$.

But for $z$ in $\Omega$ we have:

$$
\begin{aligned}
Q^{\prime}(z) & =G(z) \varphi^{\prime \prime}(z)+\psi^{\prime \prime}(z)+G^{\prime}(z) \varphi^{\prime}(z) \\
& =P(z)+G^{\prime}(z) \Phi(z) .
\end{aligned}
$$

Since $\Phi(z)$ and $Q^{\prime}(z)$ are continuous for $z$ in $\Omega \cup \gamma$, (4.4.4) extends $P(z)$ from a continuous function on $\Omega$ to a continuous function on 
$\Omega \cup \gamma$. From (4.4.2), (4.4.3) and (4.4.4) we get:

$$
2 D_{t} D_{z} u=(\alpha+i \beta)\left[P(z)+G^{\prime}(z) \Phi(z)+\varphi^{*^{\prime}}(z)\right]
$$

for $z$ on $\gamma$.

4.4.B. In a completely analogous way, we extend

$$
P^{*}(z)=z \varphi^{* \prime \prime}(z)+\psi^{* \prime \prime}(z)
$$

to a continuous function on $\hat{\Omega} \cup \gamma$, and express, for $z$ on $\gamma$,

$$
\begin{aligned}
2 D_{t} D_{\bar{z}} u= & \left(\overline{G^{\prime}(z)}\right)^{2}(\alpha-i \beta) P^{*}(z)+(\alpha+i \beta) \Phi(z) \\
& + \text { terms of order less than } 2 \text { in } \varphi^{*}(z) \text { and } \psi^{*}(z)
\end{aligned}
$$

By means of (4.3.3), (4.4.5), (4.4.6) and the fact that $\widetilde{D}_{t} D_{z}\{2 u\}=$ $D_{t} D_{z}\{2 u\}$ and $\widetilde{D}_{t} D_{\bar{z}}\{2 u\}=D_{t} D_{\bar{z}}\{2 u\}$ on $\gamma$, we have on $\gamma$ for $j+n=2$ in the notation of (4.4):

$$
\begin{aligned}
& 2 D_{x}^{j} D_{y}^{n} u=(i)^{n}\left\{P(z)+G^{\prime}(z) \Phi(z)+\varphi^{* \prime}(z)+(j-n) \varphi^{\prime}(z)\right. \\
& \left.-\frac{\alpha-i \beta}{\alpha+i \beta}\left[\varphi^{\prime}(z)+\varphi^{* \prime}(z) \overline{G^{\prime}(z)}\right]\right\} \\
& +(-i)^{n}\left\{\left(\overline{G^{\prime}(z)}\right)^{2} P^{*}(z)+\frac{\alpha+i \beta}{\alpha+i \beta} \Phi(z)+\cdots\right. \\
& \left.-\frac{\alpha+i \beta}{\alpha-i \beta} \varphi^{\prime}(z)+\cdots\right\}
\end{aligned}
$$

where $+\cdots$ means derivatives of order less than 2 in the starred terms.

\section{As seen in $\S 2$ :}

(i) $G^{\prime}(z)=(\alpha-i \beta) /(\alpha+i \beta)$ in $\Omega \cup \gamma \cup \hat{\Omega}$.

Note also that

$$
z=\overline{G[\hat{z}]}=\overline{G[\overline{G(z)}]}
$$

and therefore

$$
1=\frac{d}{d z} \overline{G[\overline{G(z)}]}=\overline{G^{\prime}(\hat{z})} G^{\prime}(z) .
$$

From this we see that

(ii) $\overline{G^{\prime}(z)}=\left[G^{\prime}(\widehat{z})\right]^{-1}$ and

(iii) $\overline{G^{\prime \prime}(z)}=-G^{\prime \prime}(\hat{z}) G^{\prime}(\widehat{z})^{-3}$

which will be needed in the expressions involving second derivations of $u$ and in equations (4.5.4).

And in particular on $\gamma$ :

(iv) $\overline{G^{\prime}(z)} G^{\prime}(z)=1$. 
Utilizing (i) and (iv) and $\varphi^{\prime}(z)=\Phi(z),(4.4 .7)$ becomes

$$
\begin{aligned}
2 D_{x}^{j} D_{y}^{n} u= & (i)^{n}\{P(z)+(j-n) \Phi(z)\} \\
& +(-i)^{n}\left\{P^{*}(z) G^{\prime}(z)^{-2}+\cdots\right\} .
\end{aligned}
$$

4.5. Inserting the above expressions for the derivatives of $u$ into the boundary conditions

$$
\begin{aligned}
& X_{n}(z)=u_{x y} \alpha(z)+u_{y y} \beta(z) \\
& Y_{n}(z)=-u_{x x} \alpha(z)-u_{x y} \beta(z)
\end{aligned}
$$

and collecting terms, we get for $z$ on $\gamma$ :

$$
\begin{aligned}
2 X_{n}(z) & =P(z)(i \alpha-\beta)+2 \Phi(z) \beta-P^{*}(z)\left[G^{\prime}(z)\right]^{-2}(i \alpha+\beta)+\cdots \\
-2 Y_{n}(z) & =P(z)(\alpha+i \beta)+2 \Phi(z) \alpha+P^{*}(z)\left[G^{\prime}(z)\right]^{-2}(\alpha-i \beta)+\cdots
\end{aligned}
$$

where $+\cdots$ signifies terms in $\varphi^{*}(z), \psi^{*}(z)$ and their derivatives of order not greater than the first. Transposing, the boundary conditions can thus be written for $z$ on $\gamma$ as:

$$
\begin{aligned}
i(\alpha+i \beta) P(z)+2 \beta \Phi(z) & =A^{*}(z) \\
(\alpha+i \beta) P(z)+2 \alpha \Phi(z) & =B^{*}(z)
\end{aligned}
$$

where

$$
\begin{aligned}
& A^{*}(z)=2 X_{n}(z)+i P^{*}(z)\left[G^{\prime}(z)\right]^{-2}(\alpha-i \beta)-(+\cdots) \\
& B^{*}(z)=-2 Y_{n}(z)-P^{*}(z)\left[G^{\prime}(z)\right]^{-2}(\alpha-i \beta)-(+\cdots)
\end{aligned}
$$

where $+\cdots$ has the same meaning as in (4.5.2). Now notice that $P^{*}(z), \varphi^{*}(z)$ and $\psi^{*}(z)$ are analytic and constructed for $z \in \hat{\Omega} \cup \gamma$, from the function $u$ and its derivatives for $z$ in $\Omega \cup \gamma$. Also notice that $P^{*}(z), \varphi^{* \prime}(z)$ and $\psi^{* \prime}(z)$ are continuous on $\hat{\Omega} \cup \gamma$. From these facts, and the hypothesis that $X_{n}(z)$ and $Y_{n}(z)$ are analytic in $\Omega \cup \gamma \cup \hat{\Omega}$ we see that $A^{*}(z)$ and $B^{*}(z)$ are known and analytic for $z$ in $\hat{\Omega}$ and continuous for $z$ in $\hat{\Omega} \cup \gamma$. Now since the determinant of (4.5.3) does not vanish for $z$ in $\Omega \cup \gamma \cup \hat{\Omega}$, we can solve for $P(z)$ and $\Phi(z)$ for $z \in \gamma$.

4.6 and 4.7. The equations (4.5.3.) analytically continue $P(z)$ and $\Phi(z)$ into $\gamma \cup \hat{\Omega}$. Since $P(z)$ and $\Phi(z)$ are known in $\Omega \cup \gamma$ by 4.4.A, we have thus obtained the analytic extension of $P(z)$ and $\Phi(z)$ into $\Omega \cup \gamma \cup \hat{\Omega}$.

4.8. To see that the analytic extension of $P(z)$ and $\Phi(z)$ into $\hat{\Omega}$ insures the analytic extension of $\varphi(z)$ and $\psi(z)$ into $\hat{\Omega}$, set, for $z \in \hat{\Omega} \cup \gamma$, $z_{0} \in \gamma$ :

$$
\varphi_{e x}(z)=\int_{z_{0}}^{z} \Phi(t) d t+\varphi\left(z_{0}\right)
$$


Then for $z$ on $\gamma, \varphi_{e x}(z)=\varphi(z)$ and thus $\varphi_{e x}(z)$ is the analytic continuation of $\varphi(z)$ into $\hat{\Omega}$.

For $z$ in $\Omega \cup \gamma \cup \hat{\Omega}$ set

$$
\Psi(z) \equiv P(z)-G(z) \Phi^{\prime \prime}(z)
$$

where we know the right hand side is analytic for $z \in \Omega \cup \gamma \cup \hat{\Omega}$. But $\Psi(z)=\psi^{\prime \prime}(z)$ for $z$ on $\Omega$ by definition (4.4.0); therefore, $\Psi(z)$ analytically continues $\psi^{\prime \prime}(z)$ into $\hat{\Omega}$. Now, for $z \in \Omega \cup \gamma \cup \hat{\Omega}, z_{0} \in \gamma$, set:

$$
\psi_{e x}(z)=\int_{z_{0}}^{z}(z-t) \Psi(t) d t+\left(z-z_{0}\right) \psi^{\prime}\left(z_{0}\right)+\psi\left(z_{0}\right)
$$

Then

$$
\psi_{e x}^{\prime \prime}(z)=\Psi(z)=\psi^{\prime \prime}(z) \text { for } z \text { in } \Omega \cup \gamma \cup \hat{\Omega}
$$

and

$$
\psi_{e x}\left(z_{0}\right)=\psi\left(z_{0}\right) \text { and } \psi_{e x}^{\prime}\left(z_{0}\right)=\psi^{\prime}\left(z_{0}\right)
$$

and thus $\psi_{e x}(z)$ analytically continues $\psi(z)$ into $\hat{\Omega}$.

Thus $\varphi(z)$ and $\psi(z)$ are analytic functions for $z \in \Omega \cup \gamma \cup \hat{\Omega}$; therefore for $z$ in $\Omega \cup \gamma \cup \hat{\Omega}$, the functions:

$$
\begin{aligned}
& \varphi_{2}^{*}(z)=\overline{\varphi(\widehat{\imath})}=\overline{\varphi[\overline{G(z)}]} \\
& \psi_{1}^{*}(z)=\overline{\psi(\widehat{z})}=\overline{\psi[\overline{G(z)}]}
\end{aligned}
$$

are analytic. Moreover $\varphi_{1}^{*}(z)$ and $\psi_{1}^{*}(z)$ agree with $\varphi^{*}(z)$ and $\psi^{*}(z)$ respectively for $z$ in $\hat{\Omega} \cup \gamma$. Thus $\varphi_{1}^{*}(z)$ and $\psi_{1}^{*}(z)$ are the analytic continuations of $\varphi^{*}(z)$ and $\psi^{*}(z)$ respectively into $\Omega \cup \gamma \cup \hat{\Omega}$; we shall drop the subscripts on $\varphi_{1}^{*}$ and $\psi_{1}^{*}$ :

Now set for $z \in \Omega \cup \gamma \cup \hat{\Omega}$.

$$
2 u_{e x}(x, y)=G(\hat{z}) \varphi(z)+z \varphi^{*}(\hat{z})+\psi(z)+\psi^{*}(\hat{z}) .
$$

Since $G(\hat{z})=\bar{z}, \varphi^{*}(\hat{z})=\overline{\varphi(z)}$ and $\psi^{*}(\hat{z})=\overline{\psi(z)}$ for $z$ in $\Omega \cup \gamma \cup \hat{\Omega}$,

$$
2 u_{e x}(x, y)=\bar{z} \varphi(z)+z \overline{\varphi(z)}+\psi(z)+\overline{\psi(z)}
$$

which is biharmonic in $\Omega \cup \gamma \cup \hat{\Omega}$. But $u_{e x}$ agrees (including derivatives) with $u(x, y)$ on $\Omega \cup \gamma$ and therefore it is the biharmonic continuation of $u(x, y)$ into $\Omega \cup \gamma \cup \hat{\Omega}$.

6. Applications to special geometrical configurations. If the function $G(z)$ is explicitly available, the previous results show that the analytic extension of $u$ in the first boundary value problem, which was just given, permits this extension expressed in terms of quadratures. 
$G(z)$ is explicitly known if $\gamma$ is explicitly given in an analytic parameter representation $z=f(t)$. Let $t=f^{-1}(z)$, then

$$
G(z)=\overline{f\left[\overline{f^{-1}(z)}\right]} \text {. }
$$

Other examples are the circle (about the origin of radius $a$ where

$$
G(z)=a^{2} z^{-1}
$$

and the ellipse $b^{2} x^{2}+a^{2} y^{2}=a^{2} b^{2}$ where

$$
G(z)=\frac{z\left(a^{2}+b^{2}\right) \pm 2 a b \sqrt{ }\left(z^{2}+b^{2}-a^{2}\right)}{a^{2}-b^{2}} .
$$

We collect the formulae which give the reflection of $u$ in terms of functions in $\Omega$, known through $u$.

From (2.1)

$$
\alpha(z)=(1 / 2)\left[\sqrt{ } G^{\prime}(z)+1 / \sqrt{ } G^{\prime}(z)\right], \quad \beta(z)=(1 / 2 i)\left[1 / \sqrt{ } G^{\prime}(z)-\sqrt{ } G^{\prime}(z)\right] .
$$

From (4.5.4)

$$
\begin{aligned}
A^{*}(z)= & 2 X_{n}(z)+\frac{i P^{*}(z)(\alpha-i \beta)}{\left[G^{\prime}(z)\right]^{2}} \\
& -\frac{i\left[z \varphi^{* \prime}(z)+\psi^{* \prime}(z)\right](\alpha-i \beta) G^{\prime \prime}(z)}{\left[G^{\prime}(z)\right]^{3}}-\frac{2 \beta \varphi^{* \prime}(z)}{G^{\prime}(z)}, \\
B^{*}(z)= & -2 Y_{n}(z)-\frac{P^{*}(z)(\alpha-i \beta)}{\left[G^{\prime}(z)\right]^{2}} \\
& -\frac{\left[z \varphi^{* \prime}(z)+\psi^{* \prime}(z)\right](\alpha-i \beta) G^{\prime \prime}(z)}{\left[G^{\prime}(z)\right]^{3}}+\frac{2 \alpha \varphi^{* \prime}(z)}{G^{\prime}(z)}
\end{aligned}
$$

We can evaluate $A^{*}(z)$ and $B^{*}(z)$ for any point $z$ in $\hat{\Omega}$ by means of (3.0), (3.1), (3.3) and (4.4B):

$$
\begin{aligned}
\varphi^{*}(z) & =\overline{\varphi(\widehat{z})}, \quad \psi^{*}(z)=\overline{\psi(\widehat{z})}, \quad P^{*}(z)=z \varphi^{* \prime \prime}(z)+\psi^{* \prime \prime}(z), \\
\varphi(z) & =(1 / 4) \int_{z_{0}}^{z}[\Delta u+i v] d t, \quad \psi_{1}(x, y)=u-\operatorname{Re}\{\bar{z} \varphi(z)\}, \\
\psi(z) & =\psi_{1}(x, y)+i \psi_{2}(x, y),
\end{aligned}
$$

once we know $G(z)$. From (4.5.3) and (2.1)

$$
\begin{aligned}
& \Phi(z)=(1 / 2) \bigvee G^{\prime}(z)\left[B^{*}(z)+i A^{*}(z)\right], \\
& P(z)=-i G^{\prime}(z)\left[\alpha(z) A^{*}(z)-\beta(z) B^{*}(z)\right] .
\end{aligned}
$$

for $z$ in $\hat{\Omega} \cup \gamma$. By means of;

$$
\varphi(z)=\int_{z_{0}}^{z} \Phi(t) d t+\varphi\left(z_{0}\right)
$$




$$
\psi(z)=\int_{z_{0}}^{z} d t(z-t)\left[P(t)-G(t) \rho^{\prime \prime}(t)\right]+\left(z-z_{0}\right) \psi^{\prime}\left(z_{0}\right)+\psi\left(z_{0}\right)
$$

we find the value of $\varphi(z)$ and $\psi(z)$ for $z$ in $\Omega$. This finally yields:

$$
2 u(x, y)=\bar{z} \varphi(z)+z \overline{\varphi(z)}+\psi(z)+\overline{\psi(z)}
$$

for $z$ in $\hat{\Omega}$.

\section{APPENDIX}

LEMMA 1. Let $g(r, \theta)$ be harmonic on the open unit disc and continuous on the closed unit disc and let $\bar{g}_{\theta}(\theta)=g_{\theta}(1, \theta)$ be continuous on the boundary of the unit disc, then $g_{\theta}(r, \theta)$ is continuous on the closed unit disc.

Proof. For $r<1$, let:

$$
g(r, \theta)=\frac{a_{0}}{2}+\sum_{n=1}^{\infty} r^{n}\left[a_{n} \cos n \theta+b_{n} \sin n \theta\right]
$$

where

$$
\left\{\begin{array}{l}
a_{n} \\
b_{n}
\end{array}\right\}=\frac{1}{\pi} \int_{-\pi}^{\pi} g(1, t)\left\{\begin{array}{l}
\cos n t \\
\sin n t
\end{array}\right\} d t
$$

Let $H[r, \theta ; \bar{g}]$ be the harmonic function of the continuous boundary values $\bar{g}$. Its Fourier coefficients are:

$$
\begin{aligned}
\left\{\begin{array}{l}
A_{n} \\
B_{n}
\end{array}\right\} & =\frac{1}{\pi} \int_{-\pi}^{\pi} \bar{g}(t)\left\{\begin{array}{l}
\cos n t \\
\sin n t
\end{array}\right\} d t \\
& =+\frac{n}{\pi} \int_{-\pi}^{\pi} g(1, t)\left\{\begin{array}{c}
\sin n t \\
-\cos n t
\end{array}\right\} d t=n\left\{\begin{array}{c}
b_{n} \\
-a_{n}
\end{array}\right\} .
\end{aligned}
$$

Hence

$$
H\left[r, \theta ; \bar{g}_{\theta}\right]=\frac{\partial}{\partial \theta} g(r, \theta)
$$$$
\text { for } r<1 \text {. }
$$

But $H\left[r, \theta, \bar{g}_{\theta}\right]$ is continuous on the closed disc $r \leqq 1$. Thus $(\partial / \partial \theta) g(r, \theta)$ is continuous in $r \leqq 1$, with boundary values $\bar{g}_{\theta}$.

Lemma 2. Let $g(r, \theta)$ be harmonic on the open unit disc $D$, be continuous on $D \cup \beta$, where $\beta$ is an open subinterval of the boundary of $D$, and let $\bar{g}_{\theta}(\theta)=g_{\theta}(1, \theta)$ be continuous on $\beta$, then $g_{\theta}(r, \theta)$ is continuous on $D \cup \beta$.

Proof. Let $\alpha$ be any subarc whose closure belongs to $\beta$. Let 
$h(\theta)$ be such that

$$
h(\theta)= \begin{cases}g(1, \theta) & \text { on } \alpha \\ 0 & \text { outside of } \beta \\ \text { in } C^{1} & \text { on } \partial D\end{cases}
$$

Let $h(r, \theta)$ be the harmonic function with $h(\theta)$ as boundary values. Consider

$$
k(r, \theta)=g(r, \theta)-h(r, \theta) \text { in } D \cup \beta .
$$

Then $k(1, \theta)=0$ on $\alpha$ and $k(r, \theta)$ is continuous on $D \cup \beta$. Since $k(1, \theta)=0$ on $\alpha$ it is analytically extensible across $\alpha$ by a well known formula. Hence $k(r, \theta)$ is continuous on and near $\alpha$. Now $g(r, \theta)=$ $h(r, \theta)+k(r, \theta)$ on $D \cup \beta$ where $h(r, \theta)$ is continuous by Lemma 1 for $0 \leqq r \leqq 1$, we find therefore $g(r, \theta)$ is continuous on $D \cup \alpha$; here $\alpha$ is an arbitrary subarc of $\beta$. Thus the result.

THEOREM. Let $f(z)=f\left(r e^{i \theta}\right)$ be analytic on the open unit disc $D$, continuous on $D \cup \beta$ (see Lemma 2 ) and let $f\left(e^{i}\right)$ be continuous on $\beta$, then $f^{\prime}(z)$ is continuously extensible into $D \cup \beta$, with $-i e^{-i \theta} f_{t}\left(e^{i \theta}\right)$ as boundary values on $\beta$.

Proof. By applying Lemma 2 to the real and imaginary parts of $f(z)$ we get that $f(z)$ is continuous on $D \cup \beta$. As $f$ is analytic on $D, f(z)=i r e^{i \theta} f^{\prime}(z)$.

COROLlaRY. If $f(z)$ is analytic on an open simply connected set $\Omega$, continuous on $\Omega \cup \gamma$, where $\gamma$ is an open analytic arc of the boundary of $\Omega$, and if the tangental derivative $f^{\prime}(z)$ is continuous on $\gamma$, then $f^{\prime}(z)$ is continuous on $\Omega \cup \gamma$.

Proof. The hypothesis and conclusion are invariant under conformal mapping, of $\Omega \cup \gamma$, hence the Theorem yields the Corollary.

\section{REFERENCES}

1. H. Lewy, On the Reflection Laws of Second Order Differential Equations in Two Independent Variables, Bull. Amer. Math. Soc., 65 (1959).

2. N. I. Muskhelishvili, Some Basic Problems of the Mathematical Theory of Elasticity, P. Noordhoff, Ltd.

3. J. M. Sloss, Reflection of Biharmonic Functions Across Analytic Boundary Conditions, Technical Report No. 17 prepared under Contract Nonr 222 (62) (NR 041-214). 



\section{PACIFIC JOURNAL OF MATHEMATICS}

\section{EDITORS}

RalPh S. Phillips

Stanford University

Stanford, California

M. G. Arsove

University of Washington

Seattle 5 , Washington
J. Dugundu

University of Southern California

Los Angeles 7, California

Lowell J. Paige

University of California

Los Angeles 24, California

\section{ASSOCIATE EDITORS}
E. F. BECKENBACH
D. DERRY
H. L. ROYDEN
E. G. STRAUS
T. M. CHERRY
M. OHTSUKA
E. SPANIER
F. WOLF

\section{SUPPORTING INSTITUTIONS}

\author{
UNIVERSITY OF BRITISH COLUMBIA \\ CALIFORNIA INSTITUTE OF TECHNOLOGY \\ UNIVERSITY OF CALIFORNIA \\ MONTANA STATE UNIVERSITY \\ UNIVERSITY OF NEVADA \\ NEW MEXICO STATE UNIVERSITY \\ OREGON STATE UNIVERSITY \\ UNIVERSITY OF OREGON \\ OSAKA UNIVERSITY \\ UNIVERSITY OF SOUTHERN CALIFORNIA
}

\author{
STANFORD UNIVERSITY \\ UNIVERSITY OF TOKYO \\ UNIVERSITY OF UTAH \\ WASHINGTON STATE UNIVERSITY \\ UNIVERSITY OF WASHINGTON \\ AMERICAN MATHEMATICAL SOCIETY \\ CALIFORNIA RESEARCH CORPORATION \\ SPACE TECHNOLOGY LABORATORIES \\ NAVAL ORDNANCE TEST STATION
}

Mathematical papers intended for publication in the Pacific Journal of Mathematrcs should be typewritten (double spaced), and the author should keep a complete copy. Manuscripts may be sent to any one of the four editors. All other communications to the editors should be addressed to the managing editor, L. J. Paige at the University of California, Los Angeles 24, California.

50 reprints per author of each article are furnished free of charge; additional copses may be obtained at cost in multiples of 50 .

The Pacific Journal of Mathematics is published quarterly, in March, June, September, and December. Effective with Volume 13 the price per volume (4 numbers) is $\$ 18.00$; single issues, $\$ 5.00$. Special price for current issues to individual faculty members of supporting institutions and to individual members of the American Mathematical Society: $\$ 8.00$ per volume; single issues $\$ 2.50$. Back numbers are available.

Subscriptions, orders for back numbers, and changes of address should be sent to Pacific Journal of Mathematics, 103 Highland Boulevard, Berkeley 8, California.

Printed at Kokusai Bunken Insatsusha (International Academic Printing Co., Ltd.), No. 6 , 2-chome, Fujimi-cho, Chiyoda-ku, Tokyo, Japan.

\section{PUBLISHED BY PACIFIC JOURNAL OF MATHEMATICS, A NON-PROFIT CORPORATION}

The Supporting Institutions listed above contribute to the cost of publication of this Journal, but they are not owners or publishers and have no responsibility for its content or policies. 


\section{Pacific Journal of Mathematics}

\section{Vol. 13, No. $4 \quad$ June, 1963}

Dallas O. Banks, Bounds for eigenvalues and generalized convexity ........... 1031

Jerrold William Bebernes, A subfunction approach to a boundary value problem for

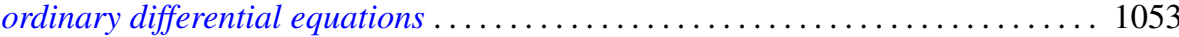

Woodrow Wilson Bledsoe and A. P. Morse, A topological measure construction . . . 1067

George Clements, Entropies of several sets of real valued functions . . . . . . . . . 1085

Sandra Barkdull Cleveland, Homomorphisms of non-commutative *-algebras . . . . . 1097

William John Andrew Culmer and William Ashton Harris, Convergent solutions of

ordinary linear homogeneous difference equations . . . . . . . . . . . . . . . 1111

Ralph DeMarr, Common fixed points for commuting contraction mappings . . . . . . 1139

James Robert Dorroh, Integral equations in normed abelian groups . . . . . . . . 1143

Adriano Mario Garsia, Entropy and singularity of infinite convolutions . . . . . . . 1159

J. J. Gergen, Francis G. Dressel and Wilbur Hallan Purcell, Jr., Convergence of extended Bernstein polynomials in the complex plane ................. 1171

Irving Leonard Glicksberg, A remark on analyticity of function algebras . . . . . . 1181

Charles John August Halberg, Jr., Semigroups of matrices defining linked operators

with different spectra ................................. 1187

Philip Hartman and Nelson Onuchic, On the asymptotic integration of ordinary

differential equations . . . . . . . . . . . . . . . . . . . . . . . . . . . . 1193

Isidore Heller, On a class of equivalent systems of linear inequalities . . . . . . . . . 1209

Joseph Hersch, The method of interior parallels applied to polygonal or multiply

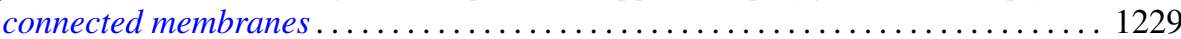

Hans F. Weinberger, An effectless cutting of a vibrating membrane . . . . . . . . . . 1239

Melvin F. Janowitz, Quantifiers and orthomodular lattices ....

Samuel Karlin and Albert Boris J. Novikoff, Generalized convex inequalities . .

Tilla Weinstein, Another conformal structure on immersed surfaces of negative

curvature.

Gregers Louis Krabbe, Spectral permanence of scalar operators

Shige Toshi Kuroda, Finite-dimensional perturbation and a representaion of

scattering operator.

Marvin David Marcus and Afton Herbert Cayford, Equality in certain

inequalities

Joseph Martin, A note on uncountably many disks .

Eugene Kay McLachlan, Extremal elements of the convex cone of semi-norms . . . . 1335

John W. Moon, An extension of Landau's theorem on tournaments . .

Louis Joel Mordell, On the integer solutions of $y(y+1)=x(x$

Kenneth Roy Mount, Some remarks on Fitting's invariants .....

Miroslav Novotný, Über Abbildungen von Mengen ............

Robert Dean Ryan, Conjugate functions in Orlicz spaces.

John Vincent Ryff, On the representation of doubly stochastic operators . . . . . . . . 1379

Donald Ray Sherbert, Banach algebras of Lipschitz functions .

James McLean Sloss, Reflection of biharmonic functions across analytic boundary

conditions with examples.

L. Bruce Treybig, Concerning homogeneity in totally ordered, connected topological space....

John Wermer, The space of real parts of a function algebra...

James Juei-Chin Yeh, Orthogonal developments of functionals and related theorems

in the Wiener space of functions of two variables......... 